Schmerz 2017· 31:620

https://doi.org/10.1007/s00482-017-0244-8

Online publiziert: 7. September 2017

(c) Deutsche Schmerzgesellschaft e.V. Published

by Springer Medizin Verlag $\mathrm{GmbH}$ - all rights reserved 2017

CrossMark

W. Eich' $\cdot$ K.-J. Bär ${ }^{2} \cdot$ M. Bernateck ${ }^{3} \cdot$ M. Burgmer ${ }^{4} \cdot$ C. Dexl ${ }^{5} \cdot$ F. Petzke ${ }^{6} \cdot$ C. Sommer ${ }^{7}$. A. Winkelmann ${ }^{8} \cdot$ W. Häuser ${ }^{9,10}$

${ }^{1}$ Abteilung Innere Medizin II (Allgemeine Innere Medizin und Psychosomatik), Universitätsklinikum Heidelberg, Heidelberg, Deutschland

${ }^{2}$ Klinik für Psychiatrie und Psychotherapie, Friedrich-Schiller-Universität Jena, Jena, Deutschland

${ }^{3}$ Zentrum für Schmerzmedizin Hannover, Hannover, Deutschland

${ }^{4}$ Klinik für Psychosomatik und Psychotherapie, Universitätsklinikum Münster, Münster, Deutschland

${ }^{5}$ Deutsche Fibromyalgie Vereinigung, Seckach, Deutschland

${ }^{6}$ Schmerzmedizin, Klinik für Anästhesiologie, Universitätsmedizin Göttingen, Göttingen, Deutschland

${ }^{7}$ Neurologische Klinik, Universitätsklinikum Würzburg, Würzburg, Deutschland

${ }^{8}$ Klinik und Poliklinik für Orthopädie, Physikalische Medizin und Rehabilitation, Klinikum der Universität München, München, Deutschland

${ }^{9}$ Innere Medizin I, Klinikum Saarbrücken gGmbH, Saarbrücken, Deutschland

${ }^{10}$ Klinik und Poliklinik für Psychosomatische Medizin und Psychotherapie, Technische Universität

München, München, Deutschland

\title{
Erratum zu: Definition, Klassifikation, klinische Diagnose und Prognose des Fibromyalgiesyndroms
}

\section{Aktualisierte Leitlinie 2017 und Übersicht von systematischen Übersichtsarbeiten}

\section{Erratum zu:}

Schmerz 2017

https://doi.org/10.1007/s00482-017-

0200-7

Die Autoren machen auf folgenden Fehler aufmerksam:

Auf S. 235, rechte Spalte, in der Box „Obligate somatische Diagnostik bei Erstevaluation" und im Kommentar S. 235-236 muss 1,25-Dihydroxy-Vitamin D durch 25-OH-Vitamin D ersetzt werden.

\section{Korrespondenzadresse}

\section{Prof. Dr. W. Eich}

Abteilung Innere Medizin II (Allgemeine Innere Medizin und Psychosomatik), Universitätsklinikum Heidelberg Im Neuenheimer Feld 410, 69210 Heidelberg, Deutschland wolfgang.eich@med.uni-heidelberg.de
Die Online-Version des Originalartikels ist unter https://doi.org/10.1007/s00482-017-0200-7 zu finden. 